\title{
ON THE ALEXANDER POLYNOMIALS OF CERTAIN THREE-COMPONENT LINKS
}

\author{
MARK E. KIDWELL
}

\begin{abstract}
Let $L$ be a three-component link all of whose linking numbers are zero. Write the Alexander polynomial of $L$ as $\Delta(x, y, z)=(1-x)(1-$ $y)(1-z) f(x, y, z)$. Then the integer $|f(1,1,1)|$ is a perfect square.
\end{abstract}

In 1953, Torres [7] gave necessary conditions for a polynomial to be the Alexander polynomial of a link. These conditions have never been proved sufficient, even though the question has appeared on at least two important lists of knot and link theory problems ([3, p. 168, no. 2], [2, p. 218, no. 15]). In this paper, we give a new condition for a polynomial to be the Alexander polynomial of a three-component link, all of whose linking numbers are zero. According to the Torres conditions, the Alexander polynomial of such a link can be written

$$
\Delta(x, y, z)=(1-x)(1-y)(1-z) f(x, y, z) .
$$

We prove that the integer $|f(1,1,1)|$ is a perfect square, and then give a class of examples to show that $|f(1,1,1)|$ can be any perfect square.

Unfortunately, there are also three-component links with one nonzero linking number whose Alexander polynomials are divisible by $(1-x)(1-$ $y)(1-z)$, and in which $|f(1,1,1)|$ is not a perfect square. Thus we have not shown that the Torres conditions are insufficient in general, but only in the presence of a restriction on the linking numbers.

The author would like to thank William Massey for his generous contribution to the discovery and proof of this theorem, and to thank the referee for raising an interesting question.

1. The main theorem. Seifert [6] gives an algorithm for spanning an orientable surface in any knot. He then represents this surface as a disk with $2 h$ bands attached, where $h$ is the genus of the surface. From this representation, he constructs the Seifert matrix, whose determinant is the Alexander polynomial.

Hosokawa [4] (as well as Torres [7]) extends this procedure to links and their reduced Alexander polynomials by adding $\mu-$ ! new bands to the above-mentioned disk and $\mu-1$ new rows and columns to the corresponding

Received by the editors June 6, 1977 and, in revised form, October 5, 1977.

AMS (MOS) subject classifications (1970). Primary 55A25.

Key words and phrases. Link, Alexander polynomial, Torres conditions, Seifert surface, Hosokawa matrix. 
matrix, where $\mu$ is the number of components in the link. (See Figure 1.) In the case of a three-component link, Hosokawa's matrix is

$$
M=\left(\begin{array}{cc|cc}
v_{1,1}(1-t) & v_{1,2}(1-t)+t \ldots & v_{1,2 h+1}(1-t) & v_{1,2 h+2}(1-t) \\
v_{1,2}(1-t)-1 & v_{2,2}(1-t) \ldots & v_{2,2 h+1}(1-t) & v_{2,2 h+2}(1-t) \\
\vdots & \vdots & \vdots & \vdots \\
\hline v_{2 h+1,1} & v_{2 h+1,2} \cdots & v_{2 h+1,2 h+1} & v_{2 h+1,2 h+2} \\
v_{2 h+2,1} & v_{2 h+2,2} \ldots & v_{2 h+2,2 h+1} & v_{2 h+2,2 h+2}
\end{array}\right)
$$

The $v_{i j}$ are overcrossing numbers of the center lines $a_{1}, \ldots, a_{2 h+\mu-1}$ of the bands in the Seifert surface. The overcrossing numbers satisfy the symmetry relations $[4$, p. 276]

$$
\begin{aligned}
v_{i, j} & =v_{j, i}=\operatorname{lk}\left(a_{i}, a_{j}\right) \quad \text { if } a_{i} \cap a_{j}=\varnothing, \\
v_{2 k-1,2 k} & =v_{2 k, 2 k-1}+1, \quad 1 \leqslant k \leqslant h .
\end{aligned}
$$

The determinant of $M$, for a three-component link, is $\Delta(t, t, t) /(1-t)$.

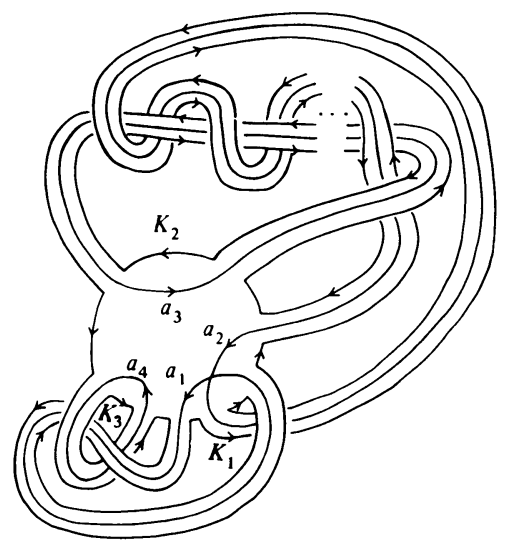

FIGURE 1

If the link $L=K_{1} \cup K_{2} \cup K_{3}$ has all its linking numbers equal to zero, then $v_{2 h+1,2 h+1}=v_{2 h+1,2 h+2}=v_{2 h+2,2 h+2}=0$. (Other overcrossing numbers, such as $v_{1,4}$ in Figure 1, can be nonzero, because both edges of the first band belong to $K_{1}$ and they are oppositely oriented.) Hence there will be a two-by-two block of zeros in the lower right-hand corner of $M$. We can then factor $(1-t)$ from the last two columns of $M$ to obtain a matrix $M^{\prime}$ whose determinant is $f(t, t, t)$.

THEOREM 1. Let $L$ be a three-component link all of whose linking numbers are zero. Write the Alexander polynomial of $L$ as $\Delta(x, y, z)=(1-x)(1-y)(1$ $-z) f(x, y, z)$. Then the integer $|f(1,1,1)|$ is a perfect square.

Proof. Replacing $t$ by 1 in the upper left-hand portion of the matrix $M^{\prime}$ changes the entries immediately above the main diagonal to +1 , those immediately below the main diagonal to -1 , and all other entries to 0 . 
Multiplying the bottom two rows by -1 produces a skew-symmetric, evendimensional matrix with integer coefficients. The determinant of such a matrix is a perfect square [1, Theorem 3.27, p. 141].

2. Examples. Figure 1 illustrates a class of three-component links with all linking numbers zero. The overcrossing number $v_{2,3}$ can be an arbitrary integer, while $v_{1,4}= \pm 1$ and $v_{1,3}=v_{2,4}=0$. The Hosokawa matrix, with factors of $(1-t)$ removed and $t$ set equal to 1 , is

$$
\left(\begin{array}{cccc}
0 & 1 & 0 & \pm 1 \\
-1 & 0 & v_{2,3} & 0 \\
0 & -v_{2,3} & 0 & 0 \\
\mp 1 & 0 & 0 & 0
\end{array}\right)
$$

Its determinant is $\pm v_{2,3}^{2}$. Thus $|f(1,1,1)|$ can be any perfect square.

The link $8_{5}^{3}$ from the table of Rolfsen and Bailey [5, p. 426] has linking numbers $( \pm 1,0,0)$ and Alexander polynomial

$$
\Delta(x, y, z)=(1-x)(1-y)(1-z)(1+x y) .
$$

Thus $|f(1,1,1)|=2$, and the restriction on linking numbers given in Theorem 1 is essential.

Addendum. The referee of this paper has reasonably asked what can be said about links of other multiplicities. If a link $L$ has $\mu \geqslant 2$ components and Hosokawa matrix $M$, then [4]

$$
\operatorname{det} M=\Delta(t, t, t) /(1-t)^{\mu-2} .
$$

If all linking numbers of $L$ are zero, there is a $(\mu-1) \times(\mu-1)$ block of zeros in the lower right-hand corner of $M$. Thus

$$
\operatorname{det} M=(1-t)^{\mu-1} \operatorname{det} M^{\prime} \text {, }
$$

where $M^{\prime}$ is obtained from $M$ by deleting factors of $(1-t)$ from the last $(\mu-1)$ columns. If $g(t)=\operatorname{det} M^{\prime}$, then $g(1)$ can again be considered the determinant of a skew symmetric integer matrix $N$. If $\mu$ is even, the dimension of $N$ is odd, and $g(1)=\operatorname{det} N=0$. [det $N=\operatorname{det}\left(N^{T}\right)=\operatorname{det}(-N)=$ $(-1)^{\operatorname{dim} N} \operatorname{det} N=-\operatorname{det} N$.] Thus $(1-t)$ divides $g(t)$. If $\mu$ is odd, the dimension of $N$ is even, and $g(1)$ is again a perfect square. Adding up factors of $(1-t)$, we have:

THEOREM 2. Let $L$ be a link of $\mu \geqslant 2$ components with all linking numbers zero. If $\mu$ is even, then $\Delta(t, \ldots, t)$ is divisible by $(1-t)^{2 \mu-2}$. If $\mu$ is odd, then $\Delta(t, \ldots, t)=(1-t)^{2 \mu-3} f(t, \ldots, t)$, where $|f(1, \ldots, 1)|$ is a perfect square.

Note that if $\mu=2$, this theorem is an immediate consequence of the Torres conditions.

\section{BIBLIOGRAPHY}

1. E. Artin, Geometric algebra, Tracts in Math., No. 3, Interscience, London, 1957.

2. J. S. Birman, Braids, links and mapping class groups, Ann. of Math. Studies, No. 82, 
Princeton Univ. Press, Princeton, N. J.; Univ. of Tokyo Press, Tokyo, 1975.

3. R. H. Fox, Some problems in knot theory, Topology of 3-Manifolds and Related Topics (Proc. Univ. of Georgia Inst., 1961), M. K. Fort (Editor), Prentice-Hall, Englewood Cliffs, N. J., 1962, pp. 168-176.

4. F. Hosokawa, D-polynomials of links, Osaka Math. J. 10 (1958), 273-282.

5. D. Rolfsen, Knots and links, Math. Lecture Ser., No. 7, Publish or Perish, Berkeley, Calif., 1976.

6. H. Seifert, Über das Geschlecht von Knoten, Math. Ann. 110 (1934-1935), 571-592.

7. G. Torres, On the Alexander polynomial, Ann. of Math. (2) 57 (1953), 57-89.

Department of Mathematics, Amherst College, Amherst, Massachusetts 01002 